\title{
Internal carotid artery dissection coincides with celiac artery dissection
}

\author{
Koen Delmotte $^{1} \cdot$ Sabrina Houthoofd $^{2} \cdot$ Philippe Demaerel $^{3} \cdot$ Robin Lemmens $^{1,4,5}$
}

Received: 29 June 2016/ Accepted: 25 July 2016

(C) Belgian Neurological Society 2016

\section{Introduction}

Spontaneous dissection of the cervical arteries is a wellrecognized cause of ischemic stroke in young and middleaged individuals. A combination of an underlying vasculopathy and an additional risk factor like a minor trauma is the proposed mechanism of spontaneous cervical artery dissections [1]. The pathophysiology of aortic dissections is commonly assumed to be different and is associated with risk factors as hypertension, old age and atherosclerosis [2]. We report a patient presenting with dissections of the carotid artery and celiac trunk within the same week.

\section{Case report}

A 53-year old man was admitted to the hospital with complaints of left sided pain in the face and neck with associated blurred vision and dizziness. There were no

Robin Lemmens

robin.lemmens@uzleuven.be

1 Department of Neurology, University Hospitals Leuven, Herestraat 49, 3000 Leuven, Belgium

2 Department of Vascular Surgery, University Hospitals Leuven, Leuven, Belgium

3 Department of Radiology, University Hospitals Leuven, Leuven, Belgium

4 Department of Neurosciences, Experimental Neurology and Leuven Research Institute for Neuroscience and Disease (LIND), KU Leuven - University of Leuven, Leuven, Belgium

5 VIB, Vesalius Research Center, Laboratory of Neurobiology, Leuven, Belgium other neurological symptoms and there was no history of a recent illness, fever or trauma.

The clinical neurological examination showed a discrete Horner syndrome on the left, but no additional deficits. A head CT (computed tomography) scan showed no abnormalities in the brain parenchyma, but a dissection of the left internal carotid artery was revealed by a CT angiography, which additionally demonstrated an aneurysmal enlargement of the right internal carotid artery, suspicious of a previous dissection (Fig. 1a, b). The patient was started on anticoagulation and discharged 2 days after admission.

Four days later he was readmitted with diffuse abdominal pains for 2 days, without fever, nausea or vomiting. On clinical examination there was some abdominal pressure pain. Laboratory tests showed mild elevation of ALT (alanine transaminase) and AST (aspartate transaminase) with normal lactate levels. An echo of the abdomen did not reveal clear abnormalities. A CT angiography of the thoraco-abdominal vessels showed a dissection of the celiac trunk, which continued into the common and right hepatic artery, with infestation of the left hepatic and the splenic artery and an intimal tear in the superior mesenteric artery (Fig. 1c, d). The patient was hospitalized for observation and strict blood pressure control while anticoagulation was continued. During the admission AST and ALT values normalized and all symptoms resolved. Therefore the patient could be discharged several days after admission.

His familial history revealed several family members with aortic aneurysms: his maternal grandfather died at the age of 76 of a thoracic aortic aneurysm and a maternal uncle had an aortic dissection at the age of 60. Mutation analysis of ACTA2, SKI, MYH11, COL3A1, FBN1, SMAD3, TGFB2, TGFBR1 and TGFBR2 to exclude genetic causes of aortic aneurysms was negative [3]. 
Fig. 1 Dissection of the internal carotid artery and celiac trunk. CT angiography of the carotid arteries (a,

b) demonstrates a dissection of the left internal carotid artery (arrow) and an aneurysmal enlargement of the right internal carotid artery (arrow head).

Thoraco-abdominal CT angiography reveales a dissection of the celiac trunk (c, arrow) with extension into the common and right hepatic artery (d, arrow)
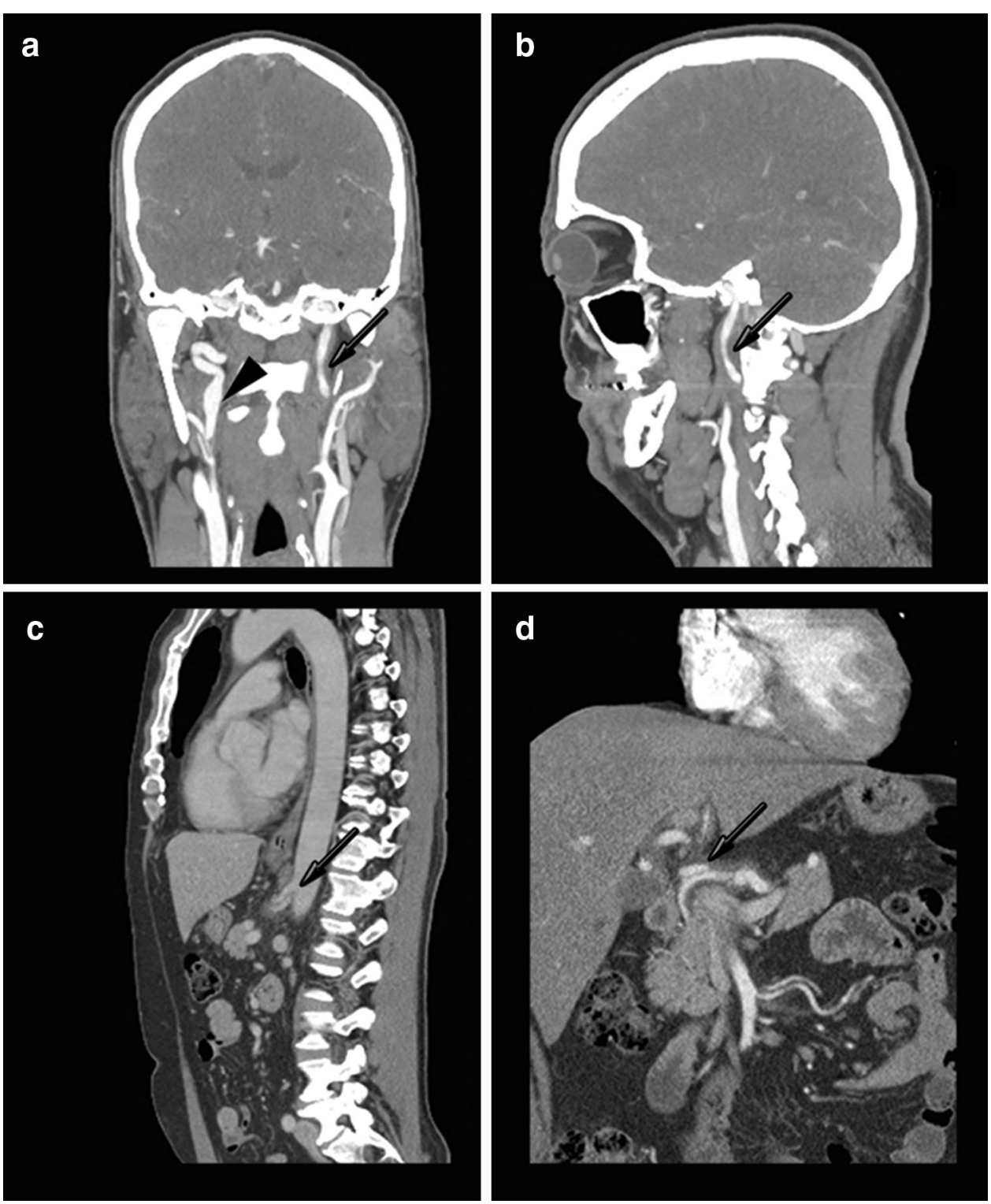

\section{Discussion}

To the best of our knowledge, this is the first case of a coincide occurrence of a carotid and celiac artery dissection. An underlying hereditary connective tissue disorder was presumed, but has not been determined.

Cervical artery dissection is a common cause of stroke in young and middle-aged patients. The presentation can vary from insidious with ipsilateral pain in the neck and face, which may be accompanied with an ipsilateral Horner's syndrome, to a severe stroke. Patients with cervical artery dissection are thought to have an underlying vasculopathy. In sporadic patients genetic and environmental risk factors, as minor cervical trauma or a recent history of an infection, are thought to act in concert resulting in the dissection. Cervical artery dissections are rarely associated with monogenetic connective tissue disorders.

Initially the pathophysiological mechanism was assumed to be an intimal tear of the cervical artery. However, more convincing evidence has shown that most likely the media and adventitia are primarily affected resulting in an intramural hematoma [4].

Spontaneous visceral dissections are very rare and are more common in men compared to women (5:1), with a mean age of approximately 55 years. Patients usually present with acute or chronic epigastric pain; and nausea, vomiting and diarrhoea can be associated. Visceral dissections can be complicated with organ failure due to ischemia or bleeding.

The pathophysiology of aortic dissections is commonly assumed to be different from cervical artery dissections. A 
combination of inherited and acquired weakening of the medial and intimal layer of the aorta is the proposed mechanism. Risk factors are hypertension, old age, atherosclerosis and previous cardiovascular surgery. Moreover, inherited disorders like Marfan's syndrome, Ehlers-Danlos syndrome type IV and other connectivetissue diseases are also associated with aortic dissections.

Treatment options for cervical artery dissection are antiplatelet versus anticoagulants. Several meta-analyses and one randomized clinical trial failed to show any significant difference in outcomes of ischemia, disability or death between the two treatment strategies [5]. Treatment for visceral dissections is even more controversial and varies from conservative follow-up, anticoagulation therapy, endovascular treatment or open surgical repair.

\section{Conclusion}

We describe a unique case of a coincide occurrence of a cervical and celiac artery dissection, in a patient with a familial history of thoracic aneurysms and dissections. A hereditary connective tissue disorder was presumed, but could not be confirmed by genetic analysis of known genes.

Acknowledgments RL is a Senior Clinical Investigator of FWO Flanders.

\section{Compliance with ethical standards}

Conflict of interest The authors declare that they have no conflict of interests.

Ethical approval The study was approved by the ethic committee of UZ Leuven of KU Leuven.

Informed consent Informed consent was obtained from the individual included in the study.

\section{References}

1. Debette S, Leys D (2009) Cervical-artery dissections: predisposing factors, diagnosis, and outcome. Lancet Neurol 8(7):668-678

2. Golledge J, Eagle KA (2008) Acute aortic dissection. Lancet 372(9632):55-66

3. Pyeritz RE (2014) Heritable thoracic aortic disorders. Curr Opin Cardiol 29(1):97-102

4. Volker W, Dittrich R, Grewe S, Nassenstein I, Csiba L, Herczeg L et al (2011) The outer arterial wall layers are primarily affected in spontaneous cervical artery dissection. Neurology 76(17):1463-1471

5. Markus HS, Hayter E, Levi C, Feldman A, Venables G, Norris J (2015) Antiplatelet treatment compared with anticoagulation treatment for cervical artery dissection (CADISS): a randomised trial. Lancet Neurol 14(4):361-367 